\title{
Carrier Spin Dephasing during Spin-Preserving Tunneling in Coupled Quantum Dots
}

\author{
M. Krzykowski*, M. GaWeeczyK and P. Machnikowski \\ Department of Theoretical Physics, Faculty of Fundamental Problems of Technology, \\ Wrocław University of Science and Technology, Wybrzeże S. Wyspiańskiego 27, 50-370 Wrocław, Poland \\ In this contribution we study carrier tunneling in a system consisting of a pair of coupled quantum dots. \\ We predict the presence of a spin dephasing channel in such a system, which is associated with a "welcher-weg" \\ type of decoherence process occurring during carrier tunneling. In our model such a process is caused by a mismatch \\ of $g$-factor values in two quantum dots in the presence of external magnetic field. This leads to a mismatch in \\ spin Zeeman splitting between the dots and, in consequence, to the distinguishability of phonons emitted during \\ the tunneling of carriers with opposite spins. Thus we demonstrate a process of spin dephasing without any direct \\ spin-environment coupling present in the model.
}

DOI: 10.12693/APhysPolA.130.1165

PACS/topics: 85.75.-d, 03.65.Yz

\section{Introduction}

Understanding of spin dynamics in a system composed of coupled quantum dots (QDs) is important due to its potential for future use in spintronic devices, like spin qubits and logic circuits [1], magnetic random access memory [2], etc. One of the key requirements to be met for a controlled use of spin states is the presence of a deterministic and coherent spin initialization scheme for a given physical system. Additionally, long life time of spin carriers is desired, which made the class of doped nanostructures of high interest and brought a proposition and realization of an optical initialization scheme for such systems [3]. The scheme, based on the optical coupling to the trion states has been, however, shown to be intrinsically partially incoherent and, moreover, to suffer from an unavoidable spin dephasing caused by the phonon bath response to the carrier excitation during the laser pulse [4]. In the view of this, systems of coupled nanostructures [5], where spin-preserving tunneling can be exploited to implement a spin-initialization scheme based on exciton dissociation [6], become a promising alternative.

In this paper we theoretically study phonon-mediated tunneling of carriers between states localized in two vertically stacked InGaAs/GaAs QDs in the presence of an in-plane magnetic field. While the spin initialization via exciton dissociation is in general a coherent process, we show in this contribution that spin coherence is not preserved during carrier tunneling in an external magnetic field if there is a misfit between $g$-factors in the QDs, which affects the fidelity of the scheme. This decoherence channel is present even in the absence of any direct coupling between the spin degree of freedom and the phonon bath. We deal here with a "welcher-weg" type of decoherence [7], where phonon bath "measures" the spin as

\footnotetext{
*corresponding author; e-mail: 204211@student.pwr.edu.pl
}

the carrier tunnels, due to the difference in the emitted phonon fields, depending on the spin orientation.

The paper is organized as follows. First, in Sect. 2 we describe our model and explain carrier dynamics therein. Then, in Sect. 3 we give a basic understanding of the nature of the "welcher-weg" type of process occurring in the system. Next, in Sect. 4 we present the results and give their discussion. Finally, we conclude the paper in Sect. 5.

\section{Model}

We model a carrier confined in a pair of vertically coupled InGaAs/GaAs QDs, assumed to be in a known initial spin state, which is practically achievable due to the optical selection rules. The system is in contact with an acoustic phonon bath via the deformation potential coupling. Additionally, the external electric field directed along the growth axis as well as magnetic field oriented perpendicularly are applied. We model the spin dynamics using a Markovian master equation in the Redfield form in a broad range of parameters, such as temperature, $g$-factors and energy levels in QDs, by solving the equation numerically.

We formulate the model in a basis of states restricted to the lowest energy states for a given location (top or bottom QD) and spin orientation, i.e. $\{|1\rangle,|2\rangle\} \otimes\{|\uparrow\rangle,|\downarrow\rangle\}$, where $|1 / 2\rangle$ corresponds to the presence of the electron in the first/second QD and $|\uparrow / \downarrow\rangle$ to the projection of its spin on the $z$ axis. For clarity and simplicity of calculations these states are modeled by Gaussians identical for both spin orientations, $\Phi(\boldsymbol{r})=N \exp \left(-\frac{x^{2}+y^{2}}{2 l^{2}}-\frac{z^{2}}{2 l_{z}^{2}}\right)$, where $l$ may serve as a measure of QD diameter, $l_{z}$ of its height, and $N$ is a normalization factor. This function is then shifted to be centered on one of the QDs, i.e. $\Phi_{1 / 2}(\boldsymbol{r})=\Phi\left(\boldsymbol{r}-\boldsymbol{r}_{1 / 2}\right)$, where $\boldsymbol{r}_{1 / 2}$ is the center of the bottom/top QD.

The Hamiltonian of the system is

$$
H=H_{c} \otimes I_{p h}+I_{c} \otimes H_{p h}+H_{c / p h},
$$


where $H_{c}$ is the carrier Hamiltonian, $H_{p h}$ is the phonon bath Hamiltonian, $H_{c / p h}$ is the carrier-phonon interaction Hamiltonian and $I_{c / p h}$ is an identity operator of rank equal to the dimension of the carrier or phonon Hilbert subspace, accordingly.

The carrier Hamiltonian $H_{c}$ is defined as $H_{c}=H_{0}+$ $H_{t}+H_{\mathrm{Z}}$, where $H_{0}=\sum_{i=1}^{2} E_{i}|i\rangle\langle i|$ is the localized carrier energy, $H_{t}=-t(|1\rangle\langle 2|+| 2\rangle\langle 1|)$ is the tunnel coupling between coupled QDs with $t$ being the coupling strength, and $\mathrm{H}_{\mathrm{Z}}$ is the Zeeman Hamiltonian depending on the external magnetic field $\boldsymbol{B}$ and the $g$-factors, $g_{i}$,

$$
H_{\mathrm{Z}}=-\frac{1}{2} \mu_{\mathrm{B}} \boldsymbol{B} \sum_{i=1}^{2} g_{i}\left[\begin{array}{cc}
\delta_{1, i} & 0 \\
0 & \delta_{2, i}
\end{array}\right] \otimes \boldsymbol{\sigma}_{i} .
$$

Here, $\boldsymbol{\sigma}_{i}$ is a vector of the Pauli matrices acting in the subspace of the $i$-th dot.

Phonon bath is introduced by a standard bosonic Hamiltonian $H_{p h}=\sum_{\boldsymbol{k}} \hbar \omega_{\boldsymbol{k}}\left(b_{\boldsymbol{k}}^{\dagger} b_{\boldsymbol{k}}+\frac{1}{2}\right)$, with $b_{\boldsymbol{k}}$ being the annihilation operator for a phonon with frequency $\omega_{\boldsymbol{k}}$. The carrier-phonon interaction Hamiltonian is

$$
H_{c / p h}=\sum_{n m, \boldsymbol{k}} F_{n m}(\boldsymbol{k}) \sigma_{n m}\left(b_{\boldsymbol{k}}+b_{-\boldsymbol{k}}^{\dagger}\right),
$$

where $F_{n m}(\boldsymbol{k})$ is the coupling constant and $\sigma_{n m}=$ $|n\rangle\langle m|$.

In further considerations we work in the basis of spatial states that diagonalizes the tunnel coupling, $\{|+\rangle,|-\rangle\}$, where $+(-)$ stands for the higher (lower) energetic state. We work in a regime of low tunnel coupling thus low spatial mixing and these states may be treated as localized in each of the QDs.

The dynamics of the system is described in the density matrix formalism by a master equation in the Redfield form [8] written in the interaction picture with respect to $H_{0}+H_{t}$ :

$$
\begin{gathered}
\dot{\rho}_{c}(t)=-\pi \sum_{l k m n} R_{l k m n}\left(\omega_{m n}\right)\left(\sigma_{l k}(t) \sigma_{m n}(t) \rho_{c}(t)\right. \\
\left.-\sigma_{m n}(t) \rho_{c}(t) \sigma_{l k}(t)\right)+ \text { H.c. }
\end{gathered}
$$

where $R_{l k m n}(\omega)$ are phonon spectral density functions describing coupling strengths,

$$
\begin{aligned}
& R_{l k m n}(\omega)= \\
& \frac{1}{\hbar^{2}(2 \pi)^{2}} \frac{\omega^{2}}{c_{1}^{3}} \int_{-1}^{1} \mathrm{~d} u F_{l k}(|\omega|, u) F_{n m}^{*}(|\omega|, u)\left|n_{\mathrm{B}}(\omega)+1\right|,
\end{aligned}
$$

where $c_{l}$ is speed of sound in the bulk material and $n_{B}$ is the Bose-Einstein distribution. The physicality of the master equation in this form may be proven by a transformation, which brings it to the Lindblad form [9].

\section{3. "Welcher-weg" type of decoherence}

In Fig. 1 we present the energy structure of the model. In the absence of the magnetic field spin states are degenerate; this degeneracy is lifted and the Zeeman splitting $\Delta E_{\mathrm{Z} \pm}$ is present in a non-zero field. In general this splitting may vary between QDs, hence the additional index $+(-)$ is referring to the orbital eigenstate.

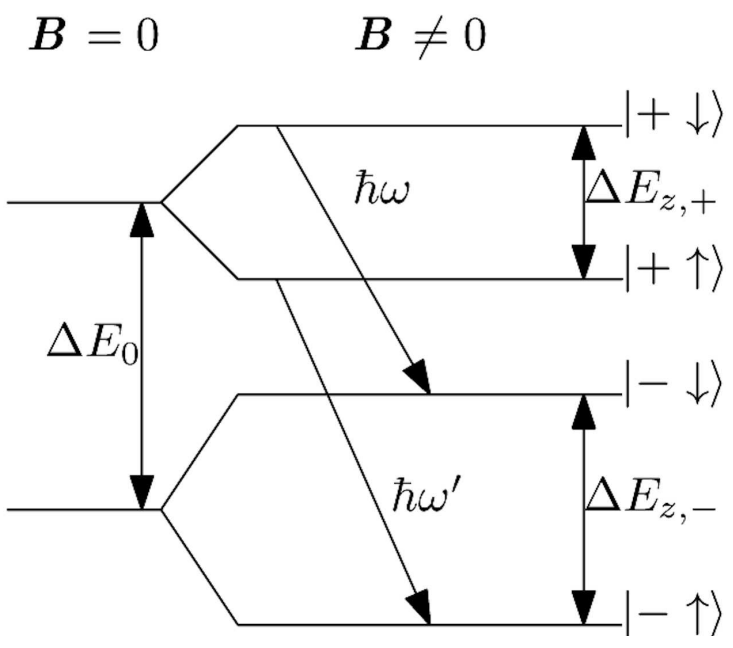

Fig. 1. Diagram of energy ladder in the model without magnetic field and with a non-zero magnetic field, where $\hbar \omega$ and $\hbar \omega^{\prime}$ denote tunnelling transition energies for the two spin orientations.

As a carrier tunnels between the states which differ in energy, a phonon is released to compensate for the excess energy. In external magnetic field, the difference in the Zeeman splittings due to the mismatch of $g$-factors in the dots causes a difference in frequency between phonons emitted during carrier tunneling between states, depending on the carrier spin orientation (see Fig. 1). Such phonons could in general be distinguished, so they carry some amount of information about the spin state of the carrier. This is the cause of the "welcher-weg" type of decoherence [7], where the phonon bath "measures" the spin of the tunneling carrier. Spin coherence after tunneling depends therefore on the frequency domain overlap of phonon wave packets, which may be a measure of the phonon distinguishability and thus of how strong the measurement of spin was.

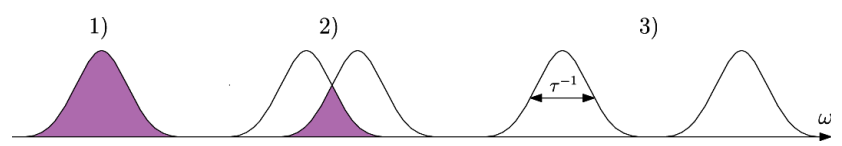

Fig. 2. Schematic showcase of phonon wave packet overlap regimes in the frequency domain.

In Fig. 2 one can find a schematic presentation of three regimes of a phonon wave packet overlap. If there is no energy mismatch (case 1) the overlap is perfect, which means full indistinguishability and there is no information transfer to the bath, hence no coherence loss. If the mismatch is small as compared to the spectral width of a packet (case 2) overlap is not full and a partial decoherence occurs. For a large mismatch (case 3) the overlap is negligible which results in a full decoherence due to a projective measurement. The spectral width of the phonon wave packet is inversely proportional to the tunneling time $\tau$. 


\section{Results}

A numerical solution of Eq. (2) gives a full information about the evolution of the system, from which we extract time dependence of electron spin polarization with respect to the $z$ axis, namely the difference between occupations of spin-up and spin-down states, as our point of interest. All results are obtained for $B=5 \mathrm{~T}$ from the initial state $\rho_{0}=|+\uparrow\rangle\langle+\uparrow|$.

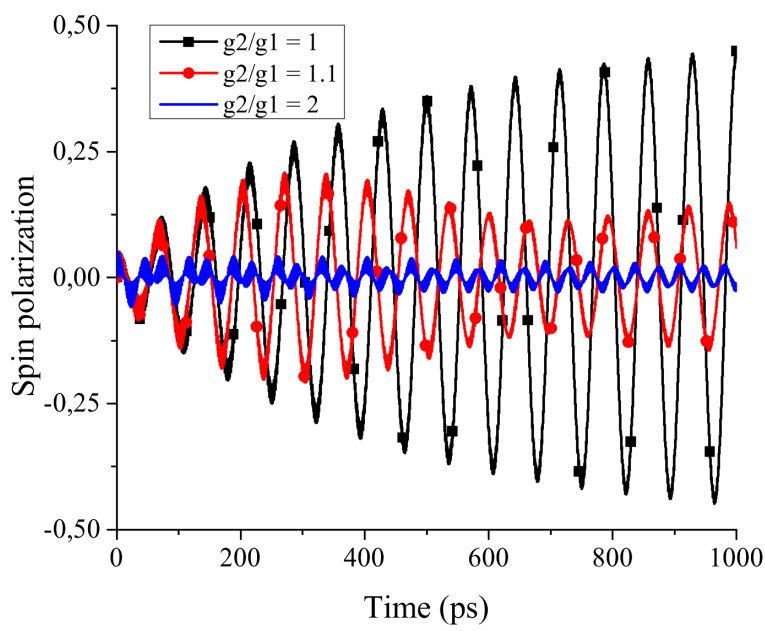

Fig. 3. Spin polarization oscillations in the second (target) QD for three values of the $g$-factor ratio: 1 (black, squares), 1.1 (red, circles), and 2 (blue) at $T=0 \mathrm{~K}$.

We begin with the presentation (Fig. 3) of the spin polarization oscillations in the target QD for the three regimes of $g$-factor mismatch described in the previous section. As expected, one observes here a fully coherent buildup of spin polarization in the case of no mismatch and a partial or full dephasing for a small or big $g$-factor ratio, respectively. The additional low-amplitude highfrequency oscillations are caused by the fact that we deal with tunnelling between states which have non-zero tails in opposite QDs.

To illustrate the dependence of the degree of discussed dephasing on the $g$-factor mismatch we plot in Fig. 4 the asymptotic value of spin coherence in the target QD as a function of the $g$-factor ratio. We find a strong correlation of the amount of coherence with the difference between $g$-factors. The process is sensitive mostly in the small $g$-factor mismatch regime, where even a small difference $(\approx 10 \%)$ results in a reduction of spin coherence by an order of magnitude. As the $g$-factor depends essentially on the QD size and composition, which are both to some extent random in the experiment, this may become an important issue from the point of view of applications.

In Fig. 5 we present the decrease of spin decoherence time with temperature. The approximately exponential decay observed here results from an accumulated coherence loss during repeated thermally activated tunnelling between the QDs which occurs at $T>0 \mathrm{~K}$.

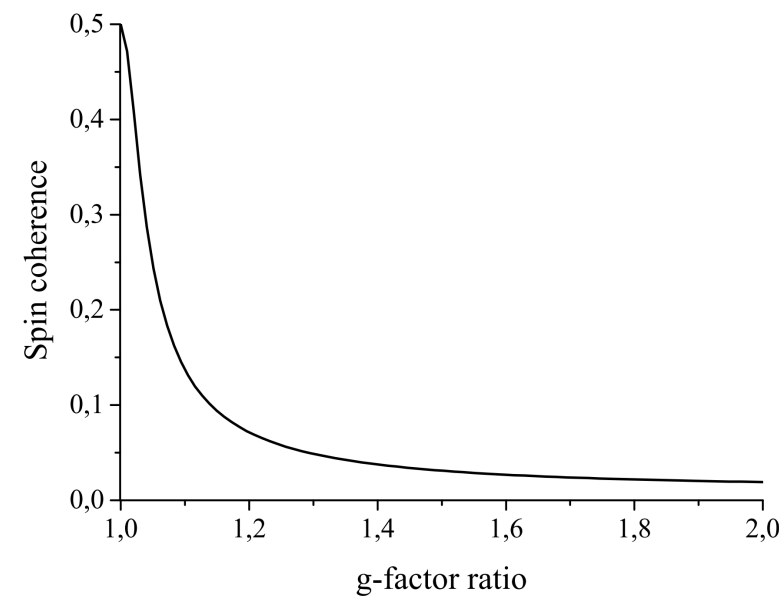

Fig. 4. Preserved spin coherence after tunnelling as function of $g$-factor ratio at $T=0 \mathrm{~K}$.

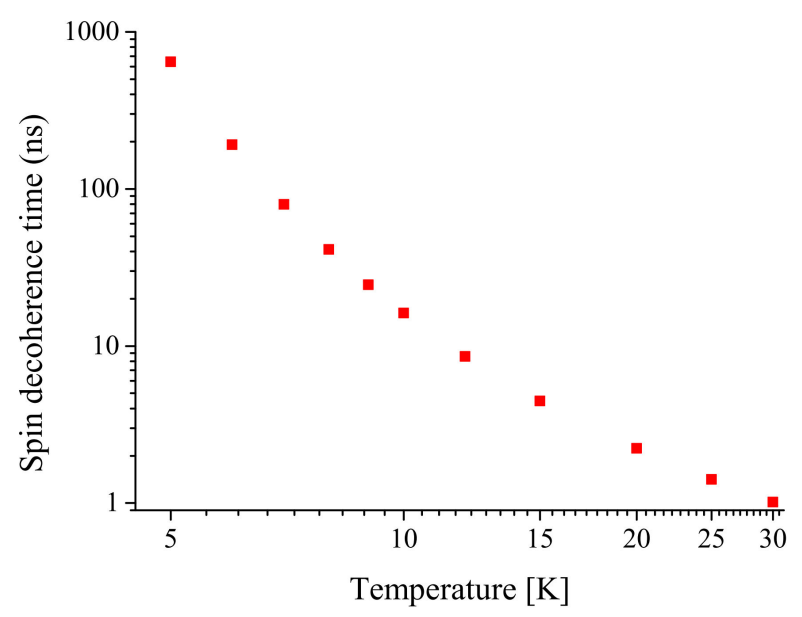

Fig. 5. Spin decoherence time as a function of temperature.

\section{Conclusions}

We have shown the presence of a "welcher-weg" type of decoherence during the tunneling of a carrier between two coupled quantum dots. The reported dephasing channel is opened due to the mismatch in the Zeeman splittings leading to the partial or full distinguishability of phonons emitted during the tunneling of carriers with opposite spin orientations. The effect is very sensitive to the $g$ factor mismatch between coupled QDs. Additionally, we have studied the impact of temperature, whose rise leads to a higher rate of back-tunneling and as a result to the accumulative dephasing.

\section{References}

[1] S. Datta, B. Das, Appl. Phys. Lett. 56, 665 (1990).

[2] W.J. Gallagher, S.S.P. Parkin, IBM J. Res. Dev. 50, 5 (2005). 
[3] M. Kugler, K. Korzekwa, P. Machnikowski, C. Gradl, S. Furthmeier, M. Griesbeck, M. Hirmer, D. Schuh, W. Wegscheider, T. Kuhn, C. Schüller, T. Korn, Phys. Rev. B 84, 085327 (2011).

[4] M. Gawełczyk, P. Machnikowski, Phys. Rev. B 87, 195315 (2013).

[5] T. Kazimierczuk, J. Suffczyński, A. Golnik, J.A. Gaj, P. Kossacki, P. Wojnar, Phys. Rev. B 79, 153301 (2009).

[6] T.M. Godden, J.H. Quilter, A.J. Ramsay, Y. Wu, P. Brereton, S.J. Boyle, I.J. Luxmoore, J. PueblaNunez, A.M. Fox, M.S. Skolnick, Phys. Rev. Lett. 108, $017402(2012)$
[7] K. Roszak, P. Machnikowski, Phys. Lett. A 73, 022313 (2006).

[8] H.-P. Breuer, F. Petruccione, The Theory of Open Quantum Systems, Oxford University Press, Oxford 2002.

[9] P. Karwat, P. Machnikowski, Phys. Rev. B 91 , 125428 (2015). 